\title{
Clinical Outcomes of Continuous Addition of Androgen Deprivation Therapy During Docetaxel Chemotherapy for Patients With Castration-Resistant Prostate Cancer
}

\begin{abstract}
Dong Hoon Lee ${ }^{1}$, Jung Ho $\mathrm{Kim}^{2}$, Won Ik Seo ${ }^{3}$, Jong Kil Nam${ }^{1}$, Tae Nam Kim , Cheol Kyu $\mathrm{Oh}^{5}$, Soo Dong Kim ${ }^{6}$, Sung-Woo Park', Jae Sung Chung ${ }^{5}$, Sang Hyun Park ${ }^{5}$, Wan Lee ${ }^{2}$, Gyung Tak Sung ${ }^{6}$, Moon Kee Chung ${ }^{1}$, Jae II Chung ${ }^{3}$

${ }^{1}$ Department of Urology, Pusan National University Yangsan Hospital, Pusan National University School of Medicine, Yangsan, ${ }^{2}$ Department of Urology, Dongnam Institute of Radiological and Medical Sciences, Busan, ${ }^{3}$ Department of Urology, Inje University Pusan Paik Hospital, Inje University College of Medicine, Busan, ${ }^{4}$ Department of Urology, Pusan National University Hospital, Pusan National University School of Medicine, Busan, ${ }^{5}$ Department of Urology, Inje University Haeundae Paik Hospital, Inje University College of Medicine, Busan, ${ }^{6}$ Department of Urology, Dong-A University College of Medicine, Busan, Korea
\end{abstract}

Purpose: This study compared the oncologic results of docetaxel chemotherapy (DOC) in castration-resistant prostate cancer (CRPC) according to continuous addition of androgen deprivation therapy (ADT) during chemotherapy.

Materials and Methods: We retrospectively reviewed the medical records of 106 patients who received DOC in 6 medical institutes. Among them, 72 patients had a complete medical record: 28 patients with ADT (DOC+continuous ADT group) and 44 without ADT (DOC only group). We compared the progression-free survival of these groups after DOC.

Results: Docetaxel was administered an average of 28 months after primary ADT as the first treatment. A median number of 6 cycles of DOC was administered in both groups. In the DOC+continuous ADT group, orchiectomy was performed in 18 patients and luteinizing hormone-releasing hormone agonist was injected in 10 patients. During DOC treatment, prostate-specific antigen (PSA) progression-free survival was statistically different $(6.0 \pm 4.75$ months in $D O C+$ continuous $A D T$ group vs. $4.8 \pm 3.2$ months in $D O C$ only group, $p=0.024)$, whereas radiologic progression-free survival was not statistically different $(5.0 \pm 3.12$ months in DOC+continuous ADT group vs. $5.0 \pm 2.79$ months in $\mathrm{DOC}$ only group, $\mathrm{p}=0.387$ )

Conclusions: In our cohort, continuous addition of ADT showed a significant benefit in PSA progression-free survival during DOC in CRPC patients. Further prospective studies are needed to confirm these observations. (Korean J Urol Oncol 2017;15:59-65)

Key Words: Androgen deprivation therapy $\cdot$ Castration-resistant prostate cancer $\cdot$ Docetaxel chemotherapy

Received April 25, 2017, Revised May 15, 2017,

Accepted May 30, 2017

Corresponding Author: Jae Il Chung

Department of Urology, Inje University Pusan Paik Hospital, Inje University College of Medicine, 75 Bokji-ro, Busanjin-gu, Busan 47392, Korea

E-mail: prosdoc@ @anmail.net

Tel: +82-51-890-6384, Fax: +82-51-892-2728

- This work was supported by 2015 clinical research grant from Pusan National University Yangsan Hospital.

\begin{abstract}
INTRODUCTION
Prostate cancer $(\mathrm{PCa})$ is the second most common cancer and the fifth leading cause of cancer-related death in males worldwide. ${ }^{1}$ In Western countries, approximately $85 \%$ of newly diagnosed PCa cases remain localized to the prostate, whereas the remainder are advanced or metastatic. ${ }^{2}$ In Korea, $21 \%$ of
\end{abstract}

(7) (7) This is an Open Access article distributed under the terms of the Creative Commons Attribution Non-Commercial License (http://creativecommons.org/licenses/by-nc/4.0/) which permits unrestricted non-commercial use, distribution, and reproduction in any medium, provided the original work is properly cited. 2017 (C) Copyright The Korean Urological Oncology Society and The Korean Prostate Society. All Rights Reserved. 
PCa patients present with distant metastases and $19 \%$ with locally advanced disease at diagnosis. ${ }^{3}$

For the advanced PCa patients, androgen deprivation therapy (ADT) has been the main treatment option. However, despite an initial favorable response, the majority of patients experience predictable resistance to ADT after 3-5 years, which is defined as castration-resistant prostate cancer (CRPC). ${ }^{4,5}$ Accumulating evidence has indicated docetaxel chemotherapy (DOC) as a standard modality after progression to CRPC. ${ }^{6,7}$ Studies have highlighted the need to maintain ADT because PCa growth remains dependent on androgen receptor signaling. ${ }^{8}$ However, it is not fully understood whether continuous addition of ADT therapy during DOC actually provides survival benefits compared with conventional DOC without ADT.

Under the current guidelines, concurrent administration of luteinizing hormone-releasing hormone (LH-RH) agonist for CRPC patients under DOC is reimbursed by the Korean National Health Insurance System. However, as mentioned above, the benefits of concurrent administration of ADT in CRPC patients under cytotoxic chemotherapy are still debatable due to the absence of tangible results from related studies. ${ }^{9,10}$

The aims of this study were therefore to examine the effect of concurrent use of ADT during DOC on clinical outcomes in Korean CRPC patients.

\section{MATERIALS AND METHODS}

This multi-institutional retrospective cohort study involved 6 hospitals in Busan and Gyeongsangnam-do, Korea. The study cohort included data from a total of 106 patients with CRPC who received DOC at the Department of Urology in each hospital between 2006 and 2016. The eligible patient population had histologically confirmed locally advanced or metastatic prostate adenocarcinoma that were treated with ADT as primary therapy between 2002 and 2015. The study population was composed of men with PCa who demonstrated disease progression as indicated by increasing prostate-specific antigen (PSA) on 2 consecutive measurements or by radiographic imaging studies. All patients were confirmed as having CRPC defined by disease progression after ADT. Before initiation of DOC, a physical examination and baseline laboratory measurements were performed. Disease status was evaluated with abdomen/pelvic computed tomography or magnetic resonance imaging and a bone scan.
After the diagnosis of CRPC, all patients received docetaxel plus prednisone therapy with or without ADT. The regimen consisted of docetaxel $\left(75 \mathrm{mg} / \mathrm{m}^{2}\right)$, which was administered through intravenous infusion once on day 1 every 3 weeks, plus oral prednisolone $5 \mathrm{mg}$ twice daily starting on day 1 and continuing throughout the treatment. DOC was continued until uncontrolled toxicity, disease progression, death, or treatment refusal by the patient. The National Cancer Institute Common Toxicity Criteria version 4.0 was used to evaluate the toxicity during each cycle. ${ }^{11}$

We excluded patients who did not have a complete medical record including PSA response and radiologic response during DOC. The final study cohort comprised 72 patients. Among them, 28 patients received concurrent ADT as maintenance during DOC (DOC+continuous ADT group) and 44 patients did not receive ADT (DOC only group).

The primary endpoints were biochemical progression-free survival (bPFS) and radiographic progression-free survival (rPFS) during DOC according to concurrent ADT use. Biochemical progression was defined as a $>50 \%$ increase from the PSA nadir, with a minimum increase of $5 \mathrm{ng} / \mathrm{mL} .^{12}$ The PSA nadir was defined as the lowest PSA level achieved during DOC. The time to biochemical progression was defined as the interval from the day of treatment initiation to biochemical progression. The time to radiographic progression was defined as the interval from the day of DOC initiation to the first occurrence of progression on imaging studies. Progression on bone scans was assessed according to the Prostate Cancer Working Group-2 criteria, ${ }^{13}$ and soft tissue progression was evaluated with reference to the Response Evaluation Criteria in Solid Tumors version 1.1. ${ }^{14}$

Differences in baseline characteristics were compared between groups using a chi-square test or Fisher exact test for categorical variables and the Mann-Whitney U-test for continuous variables. bPFS and rPFS were calculated and analyzed using the Kaplan-Meier method and the log-rank test. All of the tests were 2 -sided, and $\mathrm{p}<0.05$ was considered statistically significant. All statistical analyses were performed using IBM SPSS ver. 18.0 (IBM Co., Armonk, NY, USA).

\section{RESULTS}

Baseline patient characteristics are presented in Table 1. Both groups were statistically similar in terms of age, PSA level, 
Table 1. Baseline patient characteristics

\begin{tabular}{|c|c|c|c|}
\hline Characteristic & DOC + continuous ADT group $(n=28)$ & DOC only group $(n=44)$ & p-value \\
\hline Age at Dx (y) & $65.7 \pm 6.9$ & $65.7 \pm 8.1$ & 0.980 \\
\hline PSA at Dx (ng/dL) & $1,175 \pm 2,302$ & $446 \pm 752$ & 0.058 \\
\hline \multicolumn{4}{|l|}{ Gleason score at Dx } \\
\hline$\leq 7$ & 2 & 5 & 0.146 \\
\hline 8 & 14 & 12 & \\
\hline 9-10 & 12 & 27 & \\
\hline \multicolumn{4}{|l|}{ Clinical stage } \\
\hline cT2 & 5 & 3 & 0.348 \\
\hline cT3 & 9 & 16 & \\
\hline cT4 & 14 & 25 & \\
\hline \multicolumn{4}{|l|}{ Extent of disease } \\
\hline Bone metastasis & 19 & 34 & 0.377 \\
\hline Lymph node metastasis & 10 & 26 & 0.169 \\
\hline Liver or lung metastasis & 5 & 4 & 0.172 \\
\hline Nadir PSA after ADT $(\mathrm{ng} / \mathrm{mL})$ & $6.8 \pm 15.8$ & $10.7 \pm 31.1$ & 0.502 \\
\hline Time to PSA nadir (mo) & $8.9 \pm 7.1$ & $8.2 \pm 6.8$ & 0.705 \\
\hline Time to PSA progression (mo) & $25.8 \pm 16.1$ & $24.4 \pm 19.2$ & 0.757 \\
\hline Time to image progression (mo) & $29.0 \pm 19.2$ & $27.7 \pm 21.5$ & 0.801 \\
\hline Bone progression & 22 & 33 & 0.728 \\
\hline Lymph node progression & 2 & 7 & 0.378 \\
\hline Liver or lung progression & 3 & 8 & 0.543 \\
\hline
\end{tabular}

Values are presented as mean \pm standard deviation or number.

ADT: androgen deprivation therapy, DOC: docetaxel chemotherapy, Dx: diagnosis, PSA: prostate-specific antigen.

Table 2. Comparison of results of docetaxel chemotherapy in patients with CRPC according to concurrent ADT

\begin{tabular}{lccc}
\hline \multicolumn{1}{c}{ Variable } & DOC+continuous ADT group (n=28) & DOC only group (n=44) & p-value \\
\hline Mean Ctx cycles & 6.5 & 6.4 & 0.952 \\
Time to CTx start (mo) & $29.0 \pm 19.2$ & $27.7 \pm 21.5$ & 0.801 \\
PSA level at CTx start (ng/mL) & $126 \pm 284$ & $208 \pm 353$ & 0.301 \\
Presence of Sx at CTx start & 4 & 15 & 0.116 \\
ADT during docetaxel & & - & - \\
Orchiectomy & 18 & $4.8 \pm 3.2$ & 0.554 \\
LH-RH only & 20 & $5.0 \pm 2.79$ & 0.966 \\
Time to PSA progression (mo) & $6.0 \pm 4.75$ & 17 & 12 \\
Time to imaging progression (mo) & $5.0 \pm 3.12$ & 13 & 10 \\
Increase in old lesion & 8 & 3 & 0.005 \\
Development of new lesion & 8 & $3.4 \pm 2.55$ \\
Second-line HTx & 15 & & \\
Enzalutamide & 13 & 2 & \\
Abiraterone & $10.0 \pm 4.62$ & & \\
Time to progression after 2nd ADT (mo) & \multicolumn{3}{c}{}
\end{tabular}

Values are presented as mean \pm standard deviation or number.

CRPC: castration-resistant prostate cancer, ADT: androgen deprivation therapy, CTx: chemotherapy, Sx: symptoms, LH-RH: luteinizing hormone-releasing hormone, PSA: prostate-specific antigen, HTx: hormone therapy.

clinical stage and Gleason score, extent of metastasis at diagnosis, and biochemical/radiologic response to ADT.

Table 2 shows clinical results of DOC for the 2 groups. DOC was administered for a median number of 6 cycles in both the DOC+continuous ADT group and DOC only group. The median time to DOC after initiation of primary ADT was 
A

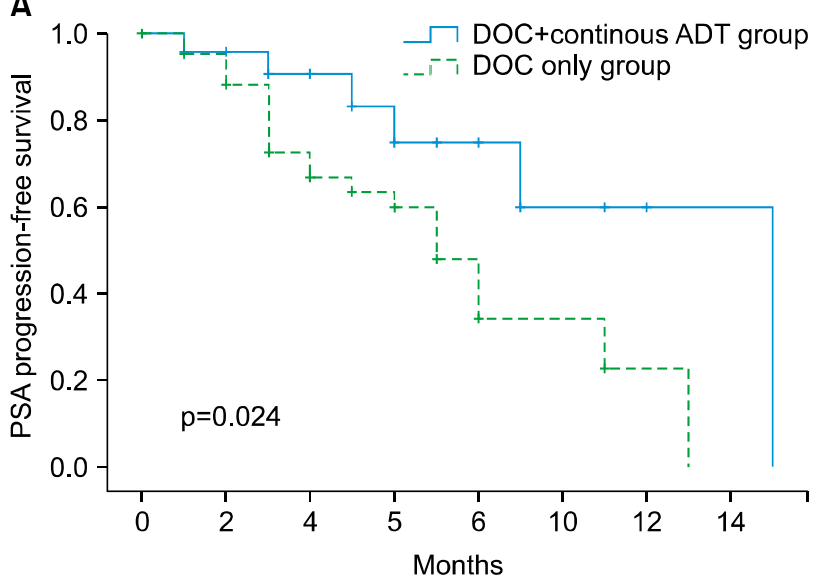

B

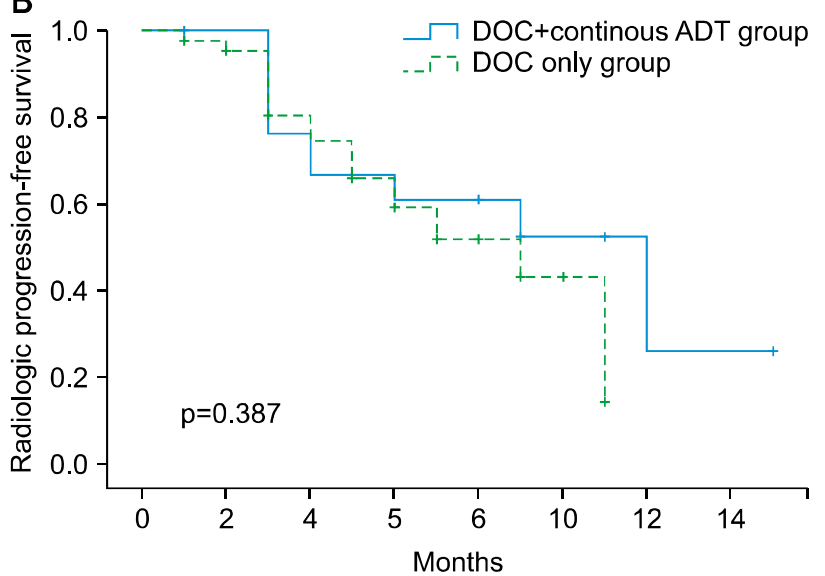

Fig. 1. Prostate-specific antigen (PSA) progression free survival and radiologic progression free survival after docetaxel chemotherapy according to concurrent androgen deprivation therapy. (A) PSA progression free survival. (B) Radiologic progression free survival. DOC: docetaxel chemotherapy, ADT: androgen deprivation therapy.

$29.0 \pm 19.2$ months and $27.7 \pm 21.5$ months, respectively.

Among the DOC+continuous ADT group ( $\mathrm{n}=28), 18$ patients underwent surgical castration (orchiectomy) and 10 patients received LH-RH agonist. Median time to biochemical progression was $6.0 \pm 4.75$ months versus $4.8 \pm 3.2$ months ( $p=0.554$ ), and time to radiologic progression was $5.0 \pm 3.12$ months versus $5.0 \pm 2.79$ months $(p=0.966)$ for the DOC+continuous ADT group versus DOC only group. The main reason for discontinuing DOC was radiologic disease progression $(57 \%$ of DOC+continuous ADT group and $52.2 \%$ of DOC only group). Other reasons included patient refusal $(n=6)$ and chemotherapy-related toxicities $(n=24)$.

Kaplan-Meier analysis revealed a significant association between concurrent administration of ADT and prolonged bPFS (log-rank p=0.024) (Fig. 1A). However, Kaplan-Meier analysis did not show a significant association between concurrent administration of ADT and prolonged rPFS (log-rank $\mathrm{p}=0.387$ ) (Fig. 1B).

\section{DISCUSSION}

DOC has been demonstrated to be efficacious and well tolerated in Western patients with advanced CRPC. ${ }^{15,16}$ This therapy was also shown to be efficacious in Korean patients with CRPC, with toxicities within acceptable limits and similar to those observed in Western patients. ${ }^{17,18}$ However, concurrent use of ADT for CRPC patients who receive DOC is a still a topic of debate. The rationale for continuing ADT when starting chemotherapy for CRPC is that cessation could lead to renewed release of testosterone and stimulation of the remaining androgen-sensitive elements of the tumor.

Regarding international guidelines, the European Association of Urology (EAU) guideline clearly states that, when CRPC develops, ADT should be continued indefinitely. ${ }^{19}$ In addition, the American Urological Association $^{20}$ and the National Comprehensive Cancer Network $^{21}$ also mention the need to maintain ADT when CRPC develops. However, the EAU guidelines are based on the data of a single study in which 85 men with CRPC refractory to orchiectomy received a chemotherapy regimen that is no longer used. $^{22}$ As a result, some investigators have doubts concerning this policy. Studies have shown that serum testosterone level remained under the castration level upon discontinuation of ADT within a certain period, and that second-line hormonal agents could replace the role of primary ADT even when disease progression was diagnosed after DOC. In fact, approximately $50 \%$ of men receiving ADT in the long term remain castrated for 2.5 years after stopping $\mathrm{ADT},{ }^{23}$ although it should be noted that stopping ADT can reinduce hormone sensitivity. ${ }^{24}$ These conflicting viewpoints are difficult to prove as there is a lack of well-designed prospective trials exploring this issue, and retrospective data are conflicting. ${ }^{25,26}$

In fact, there is no strong evidence that the combination of ADT with chemotherapy is beneficial for CRPC patients. Recently, some pivotal trials of chemotherapy in $\mathrm{PCa}$ have stipulated that ADT should be continued when chemotherapy is initiated. To date, 2 studies have compared the clinical out- 
comes of DOC according to concurrent ADT use in Korean patients. Lee et $\mathrm{al}^{26}{ }^{26}$ reported that patients treated with concurrent ADT during chemotherapy did not show significant clinical differences in progression-free survival and overall survival compared with patients who received DOC without ADT use. The proposed explanation was that serum testosterone level did not recover to the noncastrated level during the period of chemotherapy despite ADT withdrawal. However, a recent published article drew the opposite conclusion. Jang et al. ${ }^{27}$ reported that the combined use of ADT with DOC improved radiologic progression-free survival in chemotherapy-naive patients with CRPC. They suggested that concurrent administration of $\mathrm{ADT}$ and $\mathrm{DOC}$ is superior to $\mathrm{DOC}$ alone and demonstrated that concurrent administration of ADT was the only significant predictor of radiologic progression-free survival using multivariable Cox regression analysis. However, they did not show a significant difference in overall survival according to concurrent use of ADT.

In the present study, our retrospective data analysis showed that biochemical recurrence-free survival was better in CRPC patients who received DOC with concurrent ADT than in those without ADT. Unfortunately, we could not prove a significant difference in radiologic-progression free survival; however, we believe that this is due to the different time interval of laboratory and imaging examinations. When CRPC patients received DOC, we usually performed laboratory examination including PSA every month, whereas radiologic examination was performed every 3 months according to Korean insurance guidelines. Moreover, most of enrolled patients in the early period of study underwent radiologic examination when the PSA level was checked in abruptly high. This could result in statistical bias. Furthermore, this was a retrospective study using patients who were diagnosed and treated more than 10 years ago, and we believe that if we used recently improved radiologic examinations including whole spine MRI, which is more sensitive than bone scan for detection of bone progression, we might detect radiologic progression earlier in patients who had treatment without concurrent ADT than in those with concurrent ADT.

In addition, we should discuss the role of concurrent ADT in second-line hormone therapy for CRPC patients. As all randomized prospective data for abiraterone use in CRPC include the continuation of backbone ADT and O'Donnell et al. ${ }^{28}$ suggested that abiraterone monotherapy does not sustain testos- terone suppression in noncastrated men, ADT should be maintained in men with CRPC when they start abiraterone treatment. Also, all phase III trial data of enzalutamide in CRPC include the continuation of backbone ADT. This, combined with the potential adverse effects of gynecomastia with enzalutamide monotherapy, suggests that ADT should be continued when initiating enzalutamide in men with CRPC. Based on these considerations, concurrent ADT should be maintained in CRPC patients treated with hormone therapy.

A recent pivotal trial of chemotherapy, the chemo-hormonal therapy versus Androgen Ablation Randomized Trial for Extensive Disease in PCa trial, recently reported that OS was improved and metastatic load was decreased in hormone-naive men with metastatic PCa when treatment was initiated with ADT plus chemotherapy versus ADT alone. ${ }^{29}$ This is the one of the strongest reasons why concurrent ADT should be maintained in CRPC patients.

Our current study had several limitations inherent to a retrospective study including a limited number of patients. First, our data were retrospectively collected at multiple institutes, causing the results to be sensitive to selection bias. Also, concurrent ADT use was performed in mainly one center. Second, many patients were excluded due to incomplete medical records. Third, we could not show overall survival data because only a small proportion of patients died, and these patients were all from the early part of the study period. Almost all of the included patients received DOC recently. This study does not have enough power to detect the potential benefit of ADT. Finally, we could not collect serum testosterone values because it was not measured for all patients. Despite these limitations, concurrent ADT use during DOC did show a benefit in biochemical recurrence-free survival in the present study. It appears that concurrent ADT use is not beneficial in all CRPC patients. As described above, it would helpful for some patients who would experience reinduced hormone sensitivity upon stopping ADT. New and emerging clinical trials for CRPC offer the prospect of enhanced, multilayered hormonal therapies together with primary and second-line hormonal treatment, even though its effect remains unclear. Personalized/individualized management of PCa will become a reality as more treatment options/combinations that include concurrent ADT during DOC become available and are supported by clinical data. 


\section{CONCLUSIONS}

Our study showed a statistically significant advantage of ADT in biochemical progression-free survival of patients with CRPC receiving DOC. The recent approval for concurrent administration of ADT for CRPC patients treated with DOC under the present guidelines of the Korean National Health Insurance system will provide an opportunity to prove the efficacy of ADT use by comparison with clinical data collected before its approval.

\section{CONFLICT OF INTEREST}

The authors claim no conflicts of interest.

\section{REFERENCES}

1. GLOBOCAN 2012 v1.0, Cancer Incidence and Mortality Worldwide: IARC Cancer Base No. 11 [Internet]. Lyon (France): International Agency for Research on Cancer; c2013 [cited 2016 Oct 22]. Available from: http://globocan.iarc.fr

2. Ferlay J, Steliarova-Foucher E, Lortet-Tieulent J, Rosso S, Coebergh JW, Comber H, et al. Cancer incidence and mortality patterns in Europe: estimates for 40 countries in 2012. Eur J Cancer 2013;49:1374-403

3. Lee DH, Jung HB, Chung MS, Lee SH, Chung BH. The change of prostate cancer treatment in Korea: 5 year analysis of a single institution. Yonsei Med J 2013;54:87-91

4. Hinotsu S, Akaza H, Usami M, Ogawa O, Kagawa S, Kitamura $\mathrm{T}$, et al. Current status of endocrine therapy for prostate cancer in Japan analysis of primary androgen deprivation therapy on the basis of data collected by J-CaP. Jpn J Clin Oncol 2007;37:775-81

5. Cooperberg MR, Hinotsu S, Namiki M, Ito K, Broering J, Carroll PR, et al. Risk assessment among prostate cancer patients receiving primary androgen deprivation therapy. J Clin Oncol 2009;27:4306-13

6. Tannock IF, de Wit R, Berry WR, Horti J, Pluzanska A, Chi $\mathrm{KN}$, et al. Docetaxel plus prednisone or mitoxantrone plus prednisone for advanced prostate cancer. N Engl J Med 2004;351:1502-12

7. Petrylak DP, Tangen CM, Hussain MH, Lara PN Jr, Jones JA, Taplin ME, et al. Docetaxel and estramustine compared with mitoxantrone and prednisone for advanced refractory prostate cancer. N Engl J Med 2004;351:1513-20

8. Merseburger AS, Hammerer $\mathrm{P}$, Rozet $\mathrm{F}$, Roumeguère $\mathrm{T}$, Caffo O, da Silva FC, et al. Androgen deprivation therapy in castrate-resistant prostate cancer: how important is GnRH ag- onist backbone therapy? World J Urol 2015;33:1079-85

9. Taylor CD, Elson P, Trump DL. Importance of continued testicular suppression in hormone-refractory prostate cancer. J Clin Oncol 1993;11:2167-72

10. Hussain M, Wolf M, Marshall E, Crawford ED, Eisenberger M. Effects of continued androgen-deprivation therapy and other prognostic factors on response and survival in phase II chemotherapy trials for hormone-refractory prostate cancer: a Southwest Oncology Group report. J Clin Oncol 1994;12: 1868-75

11. U.S. Department of Health and Human Services, National Institutes of Health, National Cancer Institute. Common terminology criteria for adverse events (CTCAE) Ver. 4.0 [Internet]. Bethesda (MD): National Institutes of Health, National Cancer Institute (US); c2009 [updated 2010 Jun 14; cited 2015 Aug 4]. Available from: https://evs.nci.nih.gov/ ftp1/CTCAE/CTCAE_4.03_2010-06-14_QuickReference_5x7. pdf

12. Bubley GJ, Carducci M, Dahut W, Dawson N, Daliani D, Eisenberger M, et al. Eligibility and response guidelines for phase II clinical trials in androgen-independent prostate cancer: recommendations from the Prostate-Specific Antigen Working Group. J Clin Oncol 1999;17:3461-7

13. Scher HI, Halabi S, Tannock I, Morris M, Sternberg CN, Carducci MA, et al. Design and end points of clinical trials for patients with progressive prostate cancer and castrate levels of testosterone: recommendations of the Prostate Cancer Clinical Trials Working Group. J Clin Oncol 2008;26:1148-59

14. Eisenhauer EA, Therasse P, Bogaerts J, Schwartz LH, Sargent $\mathrm{D}$, Ford R, et al. New response evaluation criteria in solid tumours: revised RECIST guideline (version 1.1). Eur $\mathrm{J}$ Cancer 2009;45:228-47

15. Berthold DR, Pond GR, Soban F, de Wit R, Eisenberger M, Tannock IF. Docetaxel plus prednisone or mitoxantrone plus prednisone for advanced prostate cancer: updated survival in the TAX 327 study. J Clin Oncol 2008;26:242-5

16. Armstrong AJ, Tannock IF, de Wit R, George DJ, Eisenberger $\mathrm{M}$, Halabi S. The development of risk groups in men with metastatic castration-resistant prostate cancer based on risk factors for PSA decline and survival. Eur $\mathrm{J}$ Cancer 2010;46:517-25

17. Cho IC, Joung JY, Seo HK, Chung J, Park WS, Lee KH. Treatment outcome of docetaxel plus prednisolone for metastatic castration-resistant prostate cancer in Korea. J Cancer Res Ther 2014;10:251-7

18. Lee JL, Kim JE, Ahn JH, Lee DH, Lee J, Kim CS, et al. Efficacy and safety of docetaxel plus prednisolone chemotherapy for metastatic hormone-refractory prostate adenocarcinoma: single institutional study in Korea. Cancer Res Treat 2010;42:12-7

19. Heidenreich A, Bastian PJ, Bellmunt J, Bolla M, Joniau S, van der Kwast $\mathrm{T}$, et al. EAU guidelines on prostate cancer. 
Part II: Treatment of advanced, relapsing, and castration-resistant prostate cancer. Eur Urol 2014;65:467-79

20. Lowrance WT, Roth BJ, Kirkby E, Murad MH, Cookson MS. Castration-Resistant Prostate Cancer: AUA Guideline Amendment 2015. J Urol 2016;195:1444-52

21. Mohler JL, Armstrong AJ, Bahnson RR, D'Amico AV, Davis BJ, Eastham JA, et al. Prostate Cancer, Version 1.2016. J Natl Compr Canc Netw 2016;14:19-30

22. Manni A, Bartholomew M, Caplan R, Boucher A, Santen R, Lipton A, et al. Androgen priming and chemotherapy in advanced prostate cancer: evaluation of determinants of clinical outcome. J Clin Oncol 1988;6:1456-66

23. Bong GW, Clarke HS Jr, Hancock WC, Keane TE. Serum testosterone recovery after cessation of long-term luteinizing hormone-releasing hormone agonist in patients with prostate cancer. Urology 2008;71:1177-80

24. Shamash J, Davies A, Ansell W, Mcfaul S, Wilson P, Oliver $\mathrm{T}$, et al. A phase II study investigating the re-induction of endocrine sensitivity following chemotherapy in androgen-independent prostate cancer. Br J Cancer 2008;98:22-4
25. Taylor CD, Elson P, Trump DL. Importance of continued testicular suppression in hormone-refractory prostate cancer. J Clin Oncol 1993;11:2167-72

26. Lee JL, Eun Kim J, Ahn JH, Lee DH, Lee J, Kim CS, et al. Role of androgen deprivation treatment in patients with castration-resistant prostate cancer, receiving docetaxel-based chemotherapy. Am J Clin Oncol 2011;34:140-4

27. Jang HS, Koo KC, Cho KS, Chung BH. Survival outcomes of concurrent treatment with docetaxel and androgen deprivation therapy in metastatic castration-resistant prostate cancer. Yonsei Med J 2016;57:1070-8

28. O'Donnell A, Judson I, Dowsett M, Raynaud F, Dearnaley D, Mason M, et al. Hormonal impact of the 17alpha-hydroxylase/C(17,20)-lyase inhibitor abiraterone acetate (CB7630) in patients with prostate cancer. Br J Cancer 2004;90:2317-25

29. Sweeney CJ, Chen YH, Carducci M, Liu G, Jarrard DF, Eisenberger M, et al. Chemohormonal Therapy in Metastatic Hormone-Sensitive Prostate Cancer. N Engl J Med 2015;373: 737-46 